\title{
The effects of interval-exercise duration and intensity on oxygen consumption during treadmill running
}

\author{
Brendan J. O’Brien ${ }^{a, *}$, Jim Wibskov' ${ }^{a}$, Wade L. Knez ${ }^{\text {, }}$ \\ Carl D. Paton ${ }^{c}$, Jack T. Harvey ${ }^{a}$ \\ a School of Human Movement \& Sport Sciences, University of Ballarat, Australia \\ ${ }^{\mathrm{b}}$ Institute of Sport and Exercise Science, James Cook University, Australia \\ ${ }^{\mathrm{c}}$ Eastern Institute of Technology, New Zealand
}

\section{KEYWORDS}

Interval training;

Constant-rate training;

Endurance training;

$\dot{\mathrm{V}}_{\mathrm{O}_{2}}$

\begin{abstract}
Summary The magnitude of improvement in peak oxygen uptake $\left(\dot{V}_{\mathrm{O}_{\text {peak }}}\right)$ and performance to an exercise training regime is related to the $\dot{V}_{\mathrm{O}_{2}}$ of prior accumulated exercise training bouts. However, it is unclear whether constant rate training (CRT) or interval training (INT) preferentially alters the $\dot{V}_{\mathrm{O}_{2}}$ of running exercise. Therefore, the purpose of this study was to compare the acute $\dot{V}_{\mathrm{O}_{2}}$ response to constant, and interval training sessions. Consequently, this study compared the mean average $\dot{V}_{\mathrm{O}_{2}}$ of 17 moderately trained participants to a $20-\mathrm{min}$ CRT and two different 20 min INT treadmill runs. Participants completed three treatments (twice) in random order over 3 weeks. In 1 min INT participants completed $10 \times 1$ min efforts at the velocity corresponding to $\dot{V}_{\mathrm{O}_{2 \text { peak }}}\left(V_{\text {peak }}\right)$ interspersed with $10 \times 1 \mathrm{~min}$ efforts at $0.5 V_{\text {peak }}$. In the 2 min INT, participants completed $5 \times 2$ min efforts at the $V_{\text {peak }}$ interspersed with $5 \times 2$ min efforts at 0.5 at $V_{\text {peak }}$. In CRT participants ran at a velocity equivalent to the mean velocity of INT (75\% $\left.V_{\text {peak }}\right)$. Mean average $\dot{V}_{\mathrm{O}_{2}}$ for $2 \mathrm{~min}$ INT, $1 \mathrm{~min}$ INT and CRT were, respectively, $3200 \pm 661 ; 3076 \pm 6041 ; 2909 \pm 584 \mathrm{ml} \mathrm{min}^{-1}$. Both INT sessions resulted in a significantly higher mean average $\dot{V}_{\mathrm{O}_{2}}$ than CRT. Furthermore, 2 min INT resulted in $90 \%$ of $\dot{V}_{\mathrm{O}_{2 \text { peak }}}$ being exceeded more frequently than 1 min INT. We conclude that INT serves as a more potent stimulus for improvement in $\dot{V}_{\mathrm{O}_{\text {peak }}}$ and subsequent endurance performance than CRT.

(c) 2007 Sports Medicine Australia. Published by Elsevier Ltd. All rights reserved.
\end{abstract}

\section{Introduction}

An important determinant of maximum speed of running locomotion is the maximum metabolic power an athlete can generate over an event

\footnotetext{
Corresponding author.

E-mail address: b.obrien@ballarat.edu.au (B.J. O’Brien).
} 
distance. ${ }^{1}$ A major physiological component contributing to metabolic power and endurance performance is peak oxygen uptake $\left(\dot{V}_{\mathrm{O}_{2 \text { peak }}}\right){ }^{1}$ Therefore the improvement of $\dot{V}_{\mathrm{O}_{2 \text { peak }}}$ is an important focus of an endurance athlete's training. Consequently, athletes engage in training strategies, such as sub-maximal constant rate (CRT) and interval training (INT), to improve their $\dot{V}_{\mathrm{O}_{2 \text { peak }}}$. Unlike CRT, INT involves manipulating the peak and nadir velocity and work rest ratio of training in order to gain an advantage over CRT. Midgley and McNaughton suggest that the training strategy that results in the greatest physiological disturbance (reflected by the $\dot{V}_{\mathrm{O}_{2}}$ of the exercise task), should over a number of accumulated training sessions, produce a greater change in $\dot{V}_{\mathrm{O}_{2 \text { peak }}}{ }^{2}$. While both CRT and INT strategies may result in physiological disturbance, it remains unclear whether either strategy results in greater physiological response than the other. Additionally, it remains unclear how manipulation of INT duration and frequency affects the average $\dot{V}_{\mathrm{O}_{2}}$ during training. Knowledge of the physiological response to CRT and INT training programmes would be beneficial to athletes, as it may provide them with greater insight on the optimal strategy to enhance $\dot{V}_{\mathrm{O}_{\text {2 eak }}}$ and performance. Subsequently, the aim of this investigation was to determine the differences in average $\dot{V}_{\mathrm{O}_{2}}$ during treadmill running between CRT and INT of identical mean velocity. A second aim was to investigate how different duration and repetitions of INT affect the average $\dot{V}_{\mathrm{O}_{2}}$ and the time at which $\dot{\mathrm{V}}_{\mathrm{O}_{2}}$ exceeds $90 \%$ of the individuals $\dot{V}_{\mathrm{O}_{2 \text { peak }}}$. We hypothesize INT will have a greater mean $\dot{V}_{\mathrm{O}_{2}}$ than CRT and that $2 \mathrm{~min}$ INT will have a greater mean $\dot{V}_{\mathrm{O}_{2}}$ than 1 min INT.

\section{Methods}

\section{Subjects}

Fourteen moderately trained males and three females volunteered to participate in this study (values are expressed as mean \pm standard deviation); age: $21.9 \pm 3.9$ years, body mass: $74 \pm 11.3 \mathrm{~kg}, \quad \dot{V}_{\mathrm{O}_{2 \text { peak }}}: \quad 57.4 \pm 8.7 \mathrm{ml} \mathrm{kg}^{-1} \mathrm{~min}^{-1}$. All participants satisfied medical pre-screening criteria and gave written informed consent to participate in this experiment, which was approved by the University's Human Ethics Committee.

\section{Experimental overview}

In order to establish whether INT compared to CRT differentially affects the $\dot{V}_{\mathrm{O}_{2}}$ during treadmill run- ning, each participant completed three treatments, twice, in a balanced random order with at least $72 \mathrm{~h}$ separating treatment exposure. The three treatments were standardized for average speed over 20 min of running, and are described below:

Treatment 1: $1 \mathrm{~min}$ INT: $10 \times 1 \mathrm{~min}$ efforts at the velocity corresponding to $\dot{V}_{\mathrm{O}_{\text {2 peak }}}\left(V_{\text {peak }}\right)$ interspersed with $10 \times 1$ min efforts at $0.5 V_{\text {peak }}$.

Treatment 2: 2 min INT: $5 \times 2$ min efforts at $V_{\text {peak }}$ interspersed with $5 \times 2$ min efforts at 0.5 at $V_{\text {peak }}$.

Treatment 3: CRT: 20 min constant rate run on a treadmill at a velocity equivalent too, and determined by the mean velocity of treatment $A$ and $B(75 \%$ $V_{\text {peak }}$.

\section{Experimental procedure}

Participants initially completed a maximal treadmill test to establish $\dot{V}_{O_{2 p e a k}}$ and its corresponding velocity $\left(V_{\text {peak }}\right)$. Participants commenced running at $9 \mathrm{~km} \mathrm{~h}^{-1}$ (at a $1 \%$ gradient) and treadmill speed was increased by $1 \mathrm{~km} \mathrm{~h}^{-1}$ each minute until volitional exhaustion. Participants were fitted with a two-way breathing valve (Hans Rudolph, USA) and expired air was collected into an online metabolic system (Moxus, USA) calibrated in accordance with the manufacturers instructions. $\dot{V}_{\mathrm{O}_{2 \text { peak }}}$, was determined as the highest 60 -s $\dot{\mathrm{V}}_{\mathrm{O}_{2}}$ value recorded during the test and confirmed as a peak result if the respiratory exchange ratio (RER) exceeded 1.1.

Following the $\dot{V}_{\mathrm{O}_{2 \text { peak }}}$ test the participants were randomly assigned to one of the treatment conditions before commencing the experimental protocol. Twenty-four hours prior to each treatment, the participant was requested to eat approximately $8-10 \mathrm{~g}$ of carbohydrate per $\mathrm{kg}$ of body weight, sleep a minimum of $7 \mathrm{~h}$, and drink adequate fluid to ensure a euhydrated state. Upon reporting to the laboratory each participant completed a 5 min treadmill warm-up run at $0.6 V_{\text {peak }}$, and then rested for $3 \mathrm{~min}$. Expired air was then collected for metabolic analysis and participants began running as specified by the experimental treatment for that session. $\dot{\mathrm{O}}_{2}$ was recorded continuously in $30 \mathrm{~s}$ segments during each $20 \mathrm{~min}$ run to determine the average $\dot{V}_{\mathrm{O}_{2}}$. Time above $90 \% \dot{V}_{\mathrm{O}_{2 \text { peak }}}$ was calculated by the addition of all $30 \mathrm{~s} \dot{V}_{\mathrm{O}_{2}}$ segments recorded that exceeded $90 \%$ of the $\dot{V}_{\mathrm{O}_{2 \text { peak }}}$ of the participant. 


\section{Statistics}

Differences between the three treatments in average $\dot{V}_{\mathrm{O}_{2}}$ and $\dot{V}_{\mathrm{O}_{2}}$ expressed as a percentage of $\dot{V}_{\mathrm{O}_{2 \text { peak }}}$ (average of two trials for each treatment) were analysed in a repeated measures analysis of variance (RM ANOVA), with Bonferroni-corrected post hoc tests used to determine differences between the three treatments. Negatively skewed $\dot{V}_{\mathrm{O}_{2}}$ data were transformed to normality using a scalereversed logarithmic transformation. For percent of $\dot{V}_{\mathrm{O}_{2 \text { peak }}}$, significant negative skew occurred, but only in one group, so that a transformation to normality was not feasible; in this case the RM ANOVA The proportions of participants reaching $90 \%$ of $\dot{V}_{\mathrm{O}_{2 \text { peak }}}$ under the two treatments for which this occurred were compared using a McNemar paired samples chi-square test. All data were analysed using SPSS for Windows Version 12.0 ( ${ }^{\circledR}$ SPSS). Significance was set at $p<0.05$. Results are reported as mean \pm standard deviation (S.D.).

\section{Results}

$\dot{V}_{\mathrm{O}_{2}}$ values are presented in Table 1 . Both 2 min INT and $1 \mathrm{~min}$ INT resulted in significantly greater mean average $\dot{V}_{\mathrm{O}_{2}}$ than CRT; Mean average $\dot{V}_{\mathrm{O}_{2}}$ between 2 min INT and 1 min INT was not significantly different. A similar pattern was observed for mean average $\dot{V}_{\mathrm{O}_{2}} / \dot{V}_{\mathrm{O}_{2 \max }}(\%)$, although in this case the difference between 2 min INT and $1 \mathrm{~min}$ INT was also significant. Furthermore, a significantly greater proportion of participants reached $90 \%$ of $\dot{V}_{\mathrm{O}_{2 \text { peak }}}$ in $2 \mathrm{~min}$ INT than in $1 \mathrm{~min}$ INT $(88.2 \%$ versus $23.5 \%, p<0.001)$. Times above $90 \%$ of $\dot{V}_{\mathrm{O}_{\text {peak }}}$ (mean \pm S.D.) were $1.32 \pm 3.94 \mathrm{~min}$ and $4.47 \pm 3.55 \mathrm{~min}$, respectively. No participant exceeded $90 \%$ of $\dot{V}_{\mathrm{O}_{\text {z peak }}}$ in CRT.

\section{Discussion}

Anecdotally many endurance coaches and athletes believe that INT is more effective in improving per- formance than CRT, although there is little research to support this contention. Our findings suggests that INT may indeed be a superior mode of training to improve $\dot{V}_{\mathrm{O}_{2 \text { peak }}}$ as both INT strategies result in a significantly higher mean average $\dot{V}_{\mathrm{O}_{2}}$ than CRT and a significantly greater time spent above $90 \%$ of $\dot{V}_{\mathrm{O}_{2 \text { peak }}}$. Improvements in $\dot{V}_{\mathrm{O}_{2 \text { peak }}}$ occur as a consequence of sufficient physiological disturbance to the factors that determine it, cardiac output and arterio-venous difference. ${ }^{3}$ In particular it appears that cardiac output is the major determinant of $\dot{V}_{\mathrm{O}_{2 \text { peak }}}$ and therefore its improvement should be a major focus of endurance training. ${ }^{3}$ It appears that in order to induce the maximum change in cardiac output, training needs to extend the stroke volume to its maximum capability, which coincides with the athletes $\dot{V}_{\mathrm{O}_{2 \text { peak }}}{ }^{3}$ Therefore in order to maximise the physiological determinants that affect $\dot{V}_{\mathrm{O}_{\text {2 peak }}}$, an individual should sustain exercise training near the $\dot{V}_{\mathrm{O}_{2 \text { peak }}}$ for as long as possible. ${ }^{2}$ We observed that 2 min INT resulted in significantly more time above $90 \%$ of $\dot{V}_{\mathrm{O}_{2 \text { peak }}}$ and a significantly greater percentage of participants to exceed $90 \%$ of $\dot{V}_{\mathrm{O}_{2 \text { peak }}}$ than $1 \mathrm{~min}$ INT. Consequently, our data suggest that longer intervals may be more effective than shorter intervals of the same average intensity to enhance $\dot{V}_{\mathrm{O}_{2 \text { peak }}}$. Evidence for this contention has been provided by recent research that revealed an 8 week training regime of $15 \mathrm{~s}$ of running at $90-95 \%$ of max heart rate with $15 \mathrm{~s}$ recovery, produced smaller percentage gains in $\dot{V}_{\mathrm{O}_{2 \text { peak }}}$ than 4 min of running at $90-95 \%$ of max heart rate with 3 min recovery ( 5.5 and $7.2 \%$, respectively). ${ }^{4}$

In conclusion we report that that both interval strategies resulted in significantly higher $\dot{V}_{\mathrm{O}_{2}}$ than CRT of equal mean velocity, and that 2 min INT is more likely to result in participants to exceed $90 \%$ of $\dot{V}_{\mathrm{O}_{\text {peak }}}$ compared to $1 \mathrm{~min}$ INT. Consequently in order to maximise changes in $\dot{V}_{\mathrm{O}_{2 \text { peak }}}$ of the athlete we recommend individuals incorporate INT into their training regimes and that INT of $2 \mathrm{~min}$ duration is preferential to INT of 1 min duration.

Table 1 Mean average $\dot{V}_{\mathrm{O}_{2}}\left(\mathrm{ml} \mathrm{min}{ }^{-1}\right)$ and $\dot{V}_{\mathrm{O}_{2}} / \dot{V}_{\mathrm{O}_{2} \max }(\%)$ for the three treatments; asterisks denotes significantly different to $20 \mathrm{~min}$ CRT

\begin{tabular}{llll}
\hline & 2 min INT & 1 min INT & CRT \\
\hline Mean average, $\dot{V}_{\mathrm{O}_{2}}$ & $3200 \pm 661^{* *}$ & $3076 \pm 604^{*}$ & $2909 \pm 584$ \\
$\dot{V}_{\mathrm{O}_{2}} / \dot{V}_{\mathrm{O}_{2} \max }(\%)$ & $78.6 \pm 5.5^{* * *}, \diamond$ & $75.7 \pm 7.4^{*}$ & $71.6 \pm 5.4$ \\
\hline${ }^{*} p<0.05$. & & \\
${ }^{* *} p<0.01$. & & \\
${ }^{* * *} p<0.001$. & &
\end{tabular}


However, future research is needed to investigate the chronic effects of participating in these training strategies.

\section{References}

1. di Prampero PE. Factors limiting maximum performance in humans. Eur J Appl Physiol 2003;90(3/4): 420-9.
2. Midgley AW, McNaughton LR. Time at or near VO2max during continuous and intermittent running. A review with special reference to considerations for the optimisation of training protocols to elicit the longest time at or near V02max. $J$ Sports Med Phys Fitness 2006;46(1):1-14.

3. Basset D, Howley ET. Limiting factors for maximal oxygen uptake and determinants of endurance performance. Med Sci Sports Exerc 1998;30:1381-98.

4. Helgerud J, Hoydal K, Wang E, et al. Aerobic high-intensity intervals improve VO2max more than moderate training. Med Sci Sports Exerc 2007;39(4):665-71. 